\title{
An Algorithm for the Automatic Detection of Abnormal Mitotic Figure towards the Automated Diagnosis of Melanoma
}

\author{
Amir Parsa Anvar \\ ${ }^{a}$ School of Electrical and Electronic Engineering \\ The University of Adelaide \\ Adelaide, South Australia 5005 \\ AUSTRALIA
}

Emails: amir.p.anvar@hotmail.com; peng.shi@adelaide.edu.au; cheng.lim@adelaide.edu.au

\begin{abstract}
Histopathology is a recognised technique for the detection of melanoma, and is often referred to as the 'Gold Standard' for the detection of this disease. However, its results can sometimes be rendered inconclusive, hence demanding the need for further, more advanced, forms of testing to be conducted (e.g., FISH testing and comparative genomic hybridisation $(\mathrm{CGH})$ ). In order to support pathologists with the detection of melanoma, an algorithm has been proposed for the detection of abnormal mitotic figure; a feature of melanoma that is a key indicator of malignancy. It is anticipated that this work can contribute towards research and development in the area of automated histopathological detection of melanoma. The proposed algorithm is described here as a flow diagram. It is currently undergoing further improvement, and it will then be implemented in computer code and tested.
\end{abstract}

Keywords: Histopathology, automated abnormal mitotic figure detection, algorithm, computerised, image processing 
Amir Parsa Anvar, Peng Shi and Cheng-Chew Lim, An Algorithm for the Automatic Detection of Abnormal Mitotic Figure

\section{INTRODUCTION}

In 2011, melanoma was a part of the ten most common causes of death in Australia, ranking seventh place (AIHW, 2014). In this year, 1544 Australians died as a result of this form of cancer. In 2010, this disease was the fourth most common form of cancer to have been diagnosed in Australians (11,405 cases), after prostate cancer in males, bowel cancer and breast cancer in females (AIHW, 2014). The motivation behind this research work is to contribute to the ease of, and early, detection of this disease, which is very important for its effective treatment and, thus, the saving of lives. Therefore, a computer algorithm has been proposed for the automated detection of abnormal mitotic figure (abnormal mitosis), in histopathology images, which is a key indicator of malignancy.

If a skin lesion looks suspicious to a dermatologist, it will be biopsied and sent off to the pathologist for further examination. The pathologist will then conduct histopathological examination on the tissue. As mentioned earlier, in terms of the histopathological detection of melanoma, a key indicator for detection is abnormal mitotic figure; i.e., when a cell doesn't look like a normal dividing cell within a histopathology image. On the other hand, normal mitosis (normal mitotic figure) can be defined as when cellular division is occurring normally. As well as paying close attention towards the presence of abnormal mitoses, pathologists also count mitoses (all forms) and consider this towards their melanoma diagnosis decision. However, as mentioned earlier, the focus of this work is on the computerised detection of abnormal mitosis.

Abnormal mitotic figure can take a variety of forms. One example is tripolar mitosis. Figure 2 shows the occurrence of tripolar mitosis in a histopathology slide, which has been circled in red and indicated by the black arrow. As can be seen, tripolar mitosis exhibits a 'star-like' configuration, and is composed of three spokes that radiate from a central hub. Like tripolar mitosis, "any mitotic figure with an odd number of spokes is considered a definitive sign of malignancy" (Pathologystudent.com, 2015).

Prior to the examination of biopsy slides under the microscope, the slides are stained with Hematoxylin and Eosin. This allows for the detection of features within the tissue, and the differentiation between mitoses and other features. From the standpoint of our computer algorithm, a stained image is one that exhibits a variation of colour intensity between the pixels of abnormal mitotic figure objects and those of the rest of the image. This gives rise to the potential for intensity thresholding, allowing for the image to be separated into two classes: 1 . objects of high intensity and 2. objects of low intensity (noise, which is to be removed). Therefore, Section 2 will review thresholding. Section 2 will also review some of the work, which has been done in the field of computerised mitosis detection.

\section{RELATED WORK}

\subsection{Thresholding}

As mentioned earlier, in order to separate the abnormal mitotic figure from its background (segment it) a thresholding algorithm was necessary. Otsu's algorithm, Otsu (1979), is a well-known thresholding technique, which is adaptive (dynamic) and easily accessible via MATLAB $^{\circledR}$. In general, there are many different types of thresholding methods, and Sezgin \& Sankur (2004) have classified them into the following six areas: 1. histogram shape-based methods, 2. clustering-based methods, 3. entropy-based methods, 4. object attribute-based methods, 5. spatial methods and 6. local methods. However, due to the constraint of time, we have not been able to go into depth with the investigation of different thresholding techniques. Due to this reason, and the fact that Otsu's method met the basic criteria for solving the problem at hand, as well as its accessibility, this method was selected for application and for further investigation. Section 3 presents the results of this investigation.

\subsection{Mitosis Detection}

In terms of mitosis detection, Cireşan et al. (2013) used a Deep Neural Network-based (DNN-based) classifier to detect mitoses in breast cancer histology images. They report that the DNN is "a max-pooling (MP) convolutional neural network (CNN)", which was used as a powerful pixel classifier. Moreover, the 
Amir Parsa Anvar, Peng Shi and Cheng-Chew Lim, An Algorithm for the Automatic Detection of Abnormal Mitotic Figure

DNN "directly operates on raw RGB data sampled from a square patch of the source image, centered on the pixel itself" (Cireşan et al., 2013). In terms of the performance of their approach, Cireşan et al. (2013) achieved a precision of 0.88 , a recall of 0.70 and an $F_{1}$ score (F-measure) of 0.782 , and report that they "won the ICPR 2012 mitosis detection competition, outperforming other contestants by a significant margin". The dataset included 143 instances of mitosis for detection.

Tashk et al. (2014) worked on the detection of mitosis, by means of 2D anisotropic diffusion filtering for preprocessing, maximum likelihood estimation in the training segmentation phase, and then by extracting the completed local binary patterns of mitosis and non-mitosis candidates, object-wise. Finally, the authors report that for the classification phase, two subsequent non-linear support vector machine classifiers were trained pixel-wise and object-wise, respectively. In terms of the performance of their approach, the authors report that they achieved an $\mathrm{F}_{1}$ score of " $70.94 \%$ for Aperio XT scanner images and $70.11 \%$ for Hamamatsu images", where Aperio XT scanner images and Hamamatsu images are datasets that were assigned for the ICPR 2012 mitosis detection competition. Based upon these results, Tashk et al. (2014) have mentioned that their results indicated better performance than those of the participants of the competition.

From the short review, which has been conducted, it can be said that notable performance has been achieved in the area of mitosis detection, using powerful mathematical tools; i.e., the DNN and non-linear support vector machine classifiers. Our work differs from those works, in that it looks specifically at the detection of abnormal mitotic figure, and the detection is done by means of geometrical analysis.

\section{INVESTIGATION AND METHODOLOGY}

The proposed abnormal mitotic figure detection algorithm has two phases; the Image Preprocessing phase and the geometrical analysis phase. We have implemented the first phase in MATLAB ${ }^{\circledR}$ and are currently in the process of implementing the second phase. The first phase, along with the structure of the second phase has been described in the subsections below.

\subsection{Image Preprocessing}

This phase has one stage (conversion from RGB to binary, binarisation), however it can be thought of as having two: 1. Conversion from RGB to grayscale (Figure 3(a) to Figure 3(b)); and 2. binarisation (Figure 3(b) to 3(c)). Binarisation was done in MATLAB ${ }^{\circledR}$, with a threshold value of 0.4 .

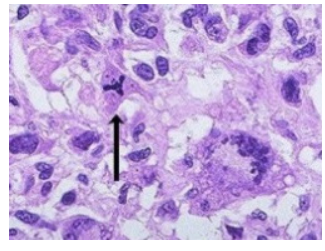

(a)

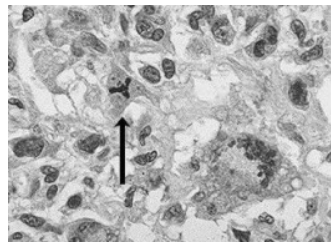

(b)

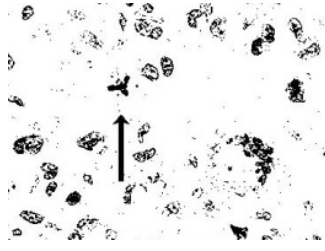

(c)

Figure 3. Image Preprocessing. (a) Original image; adapted from Pathologystudent.com (2009). (b) Grayscale image. (c) Binary image.

The results above succeeded the following investigation, which was conducted on the application of Otsu's algorithm. That is, after applying Otsu's algorithm to a number of images, it was found that the results were reasonable; however, this technique was reporting thresholds that were too high in magnitude, which meant that the separation between the two classes (high intensity pixels and low intensity noise pixels) was not as fine as we preferred it to be (See Figure 4(a)). That is, the lesser the noise surrounding the abnormal mitotic figure, the greater the chances of accurately detecting this object in the next phase of the proposed algorithm (phase 2, abnormal mitosis detection by geometrical analysis). Furthermore, it also implies that the computational load of the overall algorithm will be lighter, as the algorithm ignores white pixels for detailed assessment in phase 2, as will be seen in the next section, Section 3.2.

We have found that using a static value of 0.4 was suitable for thresholding a Hematoxylin and Eosin stained image. However, as mentioned, this approach is static and non-adaptive. In comparison, Otsu's thresholding algorithm is an automatic technique; i.e., is adaptive to different cases (images). Therefore, our aim for the future is to create an automatic thresholding technique that generates low thresholds. 
Amir Parsa Anvar, Peng Shi and Cheng-Chew Lim, An Algorithm for the Automatic Detection of Abnormal Mitotic Figure

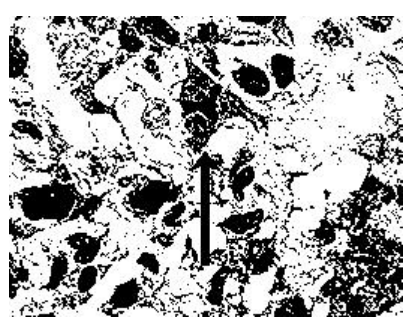

(a)

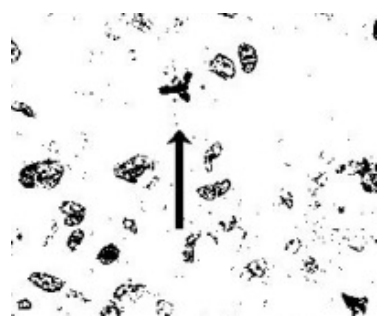

(b)

Figure 4. The result of segmenting the original image with (a) Otsu's threshold. (b) a threshold value of 0.4. The 3-spoke abnormal mitotic figure (star-like object) is the target for detection. Original image adapted from Pathologystudent.com (2009).

\subsection{Phase 2, Stage 1: Pixel Location Nomination}

The first stage of the geometrical assessment phase of the proposed abnormal mitotic figure detection algorithm is referred to as 'pixel location nomination'. The purpose of this stage is to evaluate whether a particular pixel location is suitable for further analysis; i.e., for geometrical analysis. Figure 5 explains the procedure for this stage. As shown, it comprises a series of conditions, within a loop (the dark yellow hexagon), which investigate the position of the current pixel, with respect to the locations of the edges of the image, to determine whether the pixel is within bounds. They also investigate the situation of the current pixel and its neighbours, in terms of binary value (colour), to judge whether further analysis can be done. The boundary checking operation is still under investigation, and thus has been assigned for future work. The reason behind determining whether the current pixel and its neighbours are black in colour, is to ensure that there exists a foundation for further search; i.e., essentially a 'dark block', which has potential for further investigation.

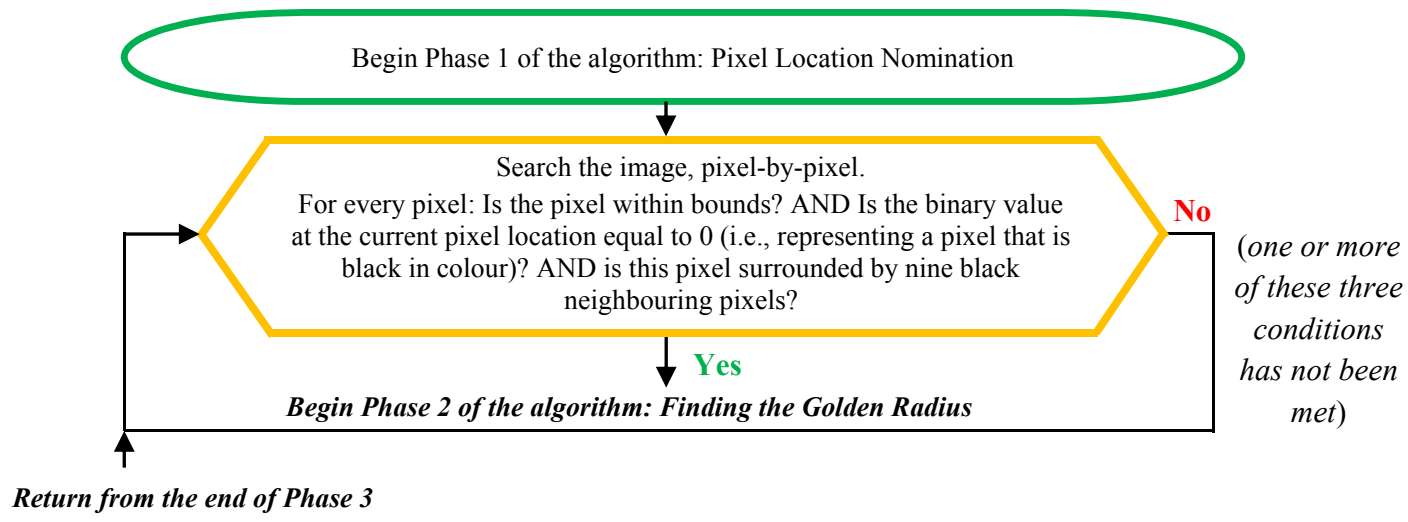

Figure 5. The flow diagram for Stage 1 of Phase 2 of the proposed abnormal mitotic figure detection algorithm: Pixel Location Nomination.

\subsection{Phase 2, Stage 2: Finding the Golden Radius}

The 'golden radius' is defined as the radius from the nominated pixel to a point that is acceptable, in order for the angular search in the next stage of the algorithm (Bitflip Analysis for Abnormal Mitotic Figure Detection) to be conducted effectively. Figure 6 depicts this radius. This figure and Equation (1) show the constraints on the size of this radius and describe the form that it takes. The procedure for Stage 2 is shown in Figure 7.

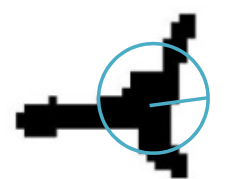

Figure 6. An illustration of the golden radius.

goldenradius $=\frac{\text { shortest of the three longest radii }+ \text { longest of the three shortest radii }}{2}$ 
Amir Parsa Anvar, Peng Shi and Cheng-Chew Lim, An Algorithm for the Automatic Detection of Abnormal Mitotic Figure

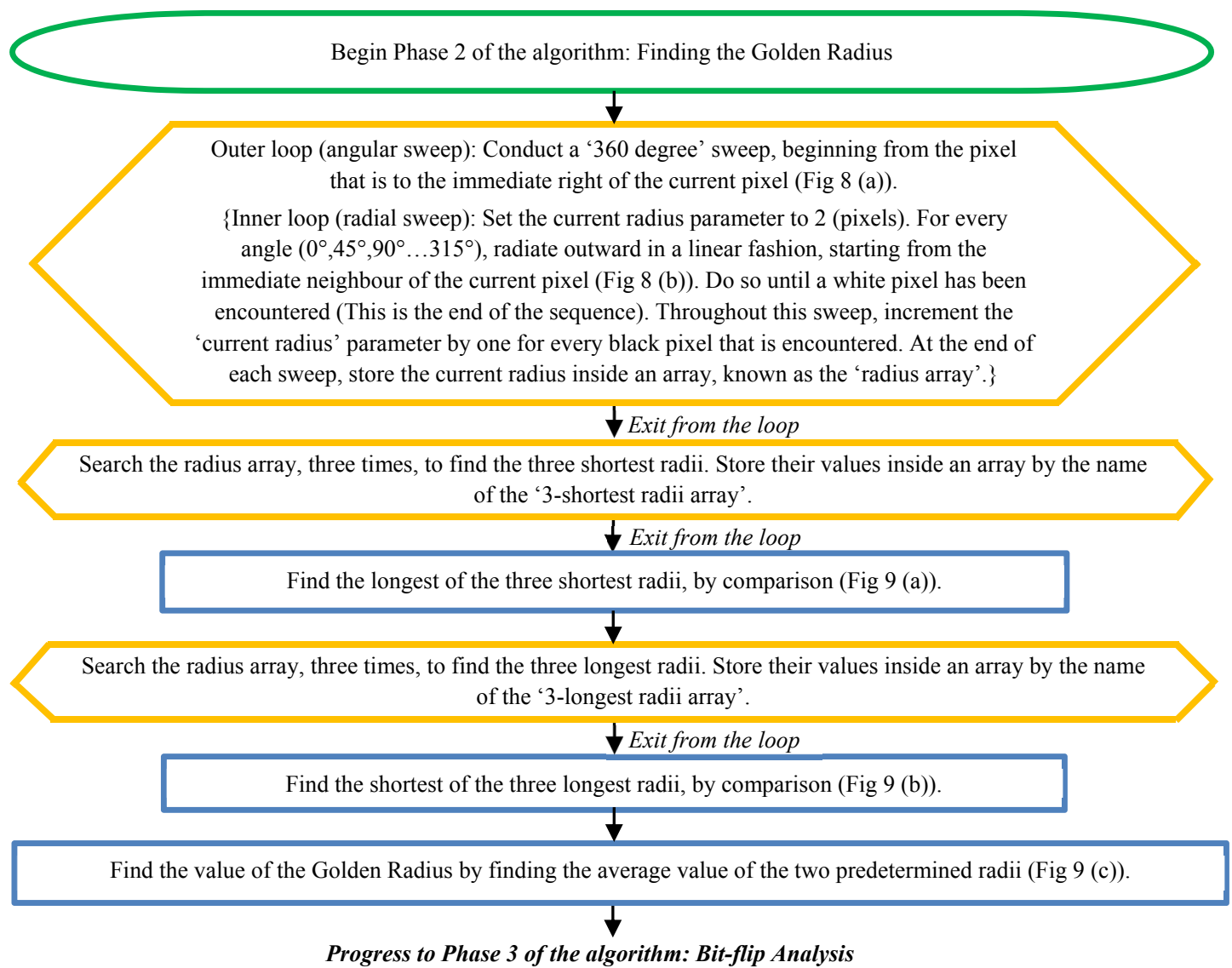

Figure 7. The flow diagram for Stage 2 of Phase 2 of the proposed abnormal mitotic figure detection algorithm: Finding the Golden Radius.

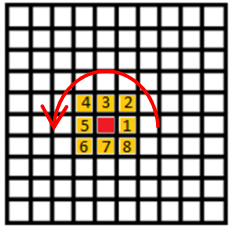

(a)

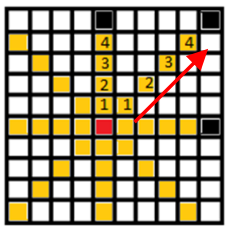

(b)

Figure 8. An illustration of the two intertwined search procedures (a) the ' 360 degree' sweep. (b) the outward radiating linear sweep.

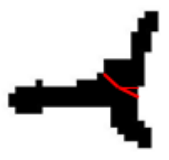

(a)

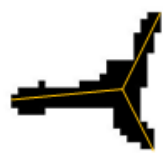

(b)

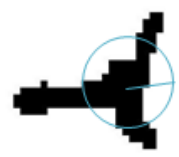

(c)

Figure 9. (a) Step 1: Finding the longest of the three shortest radii. (b) Step 2: Finding the shortest of the three longest radii. (c) Step 3: Finding the average radius (the golden radius).

\subsection{Phase 2, Stage3: Bit-flip Analysis for Abnormal Mitotic Figure Detection}

This operation is essentially a spoke counter. The working principle behind this stage has been illustrated in Figure 10, and is described in the flow diagram shown below (Figure 11). Basically, an edge is noted every time a bit flips within the search pattern. At the end of the algorithm, Equation (2) is used to calculate the number of spokes from the number of edges, and the number of spokes is then used to judge if an abnormal mitotic figure is present.

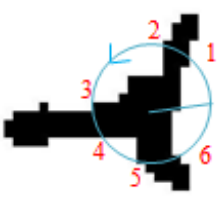

Figure 10.

An illustration of bit-flip analysis for abnormal mitotic figure detection. 
Amir Parsa Anvar, Peng Shi and Cheng-Chew Lim, An Algorithm for the Automatic Detection of Abnormal Mitotic Figure

SpokeCount $=\frac{\text { Number of edges (the number of bit flips) }}{2}$

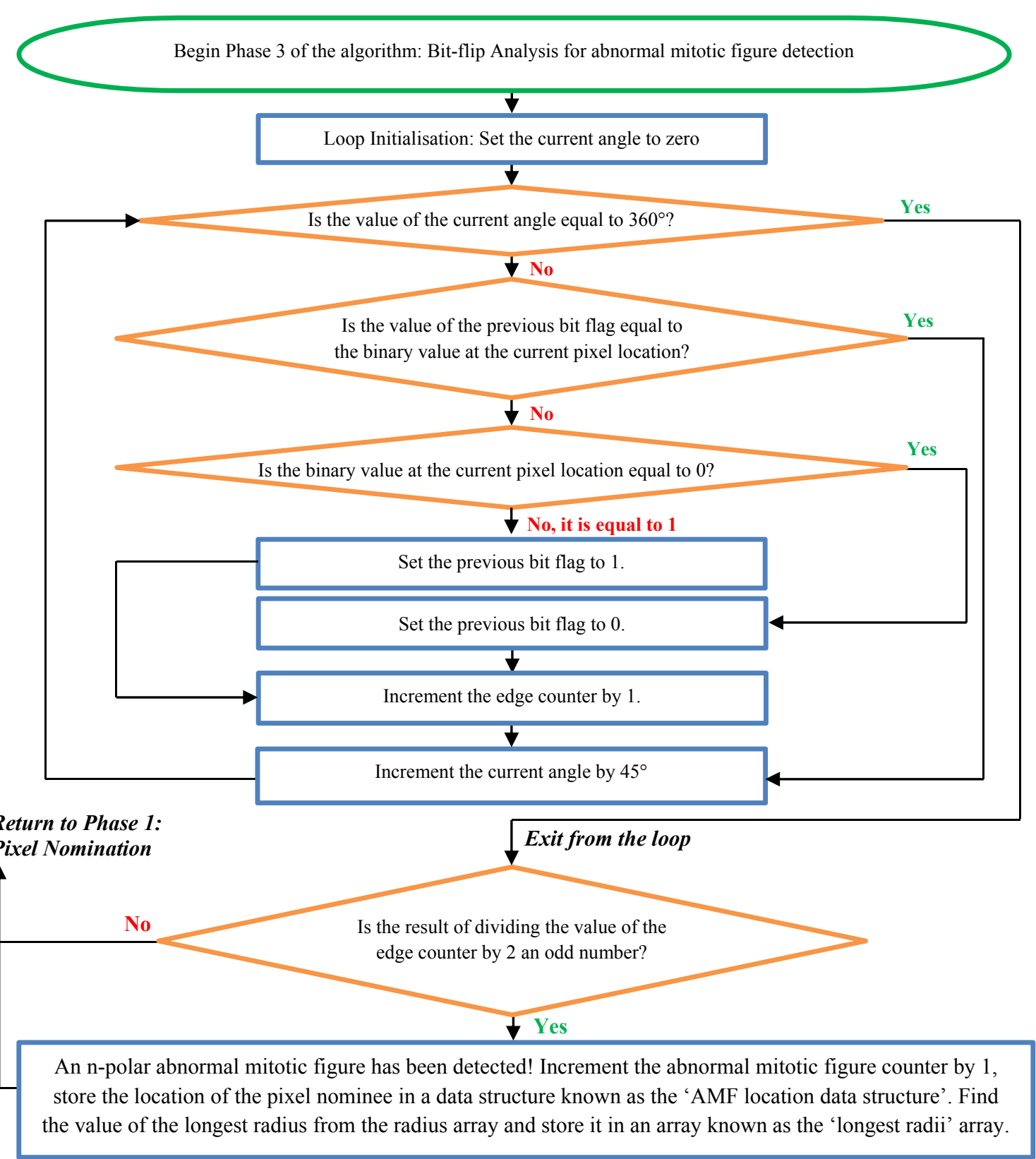

Figure 11. The flow diagram for Stage 3 of Phase 2 of the proposed abnormal mitotic figure detection algorithm: Bit-flip Analysis for Abnormal Mitotic Figure Detection.

At the end of the algorithm, a circle will be drawn around every detected abnormal mitotic figure, based upon the values in the AMF location data structure and the longest radii array.

\section{DISCUSSION AND CONCLUSIONS}

The aim of this work is to devise an algorithm for the automated detection of abnormal mitotic figure. This aim has been partly accomplished, by means of the creation of a flow diagram for a 2-phase algorithm. In the first phase of this algorithm (Image Pre-processing), the image is binarised to increase the chances of detecting abnormal mitotic figures, and to allow for their faster detection. In the second phase, which has a number of stages, the pixels in the image are analysed, based upon their binary values (colours), and an attempt is ultimately made to identify the presence of abnormal mitotic figure by examining the number of 
Amir Parsa Anvar, Peng Shi and Cheng-Chew Lim, An Algorithm for the Automatic Detection of Abnormal Mitotic Figure

detected spokes. This comprehensive design appears to be well-grounded, and is in the process of undergoing further improvement. It is then intended for the algorithm to be implemented in computer code and tested.

\section{FUTURE WORK}

Once the algorithm has been implemented, performance measures such as Precision, Recall (Sensitivity or True Positive Rate), Accuracy and F-measure will be used to validate it.

As mentioned in Section 3.1, after applying Otsu's algorithm to a number of images, it was found that this technique was reporting thresholds that were too high in value. Therefore, our aim for the future, within this area, is to create an automatic thresholding technique that generates lower thresholds, which satisfy the design requirements.

Moreover, the current algorithm can only detect abnormal mitotic figure objects that reside within the bounds of the image, and not those that lie out of bounds. This case must be addressed. Another problem, which must be addressed, is in relation to the recording of the values of the radii that surround the nominated pixel. During this phase, it can occur for a radius to take a length that is too long; i.e., a length that is absurdly long. Therefore, sensible constraints must be applied to handle this case. Also, a bit-flip does not always imply the occurrence of an edge. Bit-flips can occur due to the presence of unwanted noise. This exception can be handled by determining if there exists a radius at the angle where each bit-flip occurs. Finally, the current design inflates the abnormal mitotic figure count a multiple number of times per abnormal mitotic figure, which is undesirable. We intend to calculate the centroid, surrounding the nominated pixel location, with respect to limits that have been calculated based upon the value of the longest radius, towards the solution to this problem.

\section{ACKNOWLEDGEMENTS}

We would like to thank Dr Craig James, from Adelaide Pathology Partners, Mr Jim Manavis, from The University of Adelaide, Dr Catherine Reid, from St Peters Dermatology Clinic, Associate Professor Pascale Guitera, from Sydney University, and Dr Jeffrey Wayte, from Dermatology SA, for their invaluable guidance, suggestions and support.

\section{REFERENCES}

AIHW (Australian Institute of Health and Welfare). (2014). Australia's health 2014: The 14th

biennial health report of the Australian Institute of Health and Welfare.

http://www.aihw.gov.au/WorkArea/DownloadAsset.aspx?id=60129548150

Cireşan, D. C., Giusti, A., Gambardella, L. M., \& Schmidhuber, J. (2013). Mitosis detection in breast cancer histology images with deep neural networks. In Medical Image Computing and Computer-Assisted Intervention-MICCAI 2013 (pp. 411-418). Springer Berlin Heidelberg.

Otsu, N. (1979). A threshold selection method from gray-level histograms. IEEE Transactions on Systems, Man and Cybernetics, 9(1), 62-66.

Pathologystudent.com,. (2009). anaplasia. http://www.pathologystudent.com/?attachment_id=835

Pathologystudent.com,. (2015). How do you identify mitoses?. http://www.pathologystudent.com/?p=5689

Sezgin, M. (2004). Survey over image thresholding techniques and quantitative performance evaluation. Journal of Electronic Imaging, 13(1), 146-168.

Soyer, H. P., Argenziano, G., Hofmann-Wellenhof, R., \& Zalaudek, I. (2011). Dermoscopy: The Essentials, 2nd edition. Elsevier Health Sciences.

Tashk, A., Helfroush, M. S., Danyali, H., \& Akbarzadeh, M. (2014). A novel CAD system for mitosis detection using histopathology slide images. Journal of Medical Signals and Sensors, 4(2), 139-149. 